Check for updates

Cite this: RSC Adv., 2019, 9, 1726

Received 20th October 2018

Accepted 7th January 2019

DOI: $10.1039 / c 8 r a 08694 j$

rsc.li/rsc-advances

\section{Influence of magnetic field on electrical and thermal transport in the hole doped ferromagnetic manganite: $\mathrm{La}_{0.9} \mathrm{Na}_{0.1} \mathrm{MnO}_{3}$}

\author{
Rajasree Das, Amit Chanda and Ramanathan Mahendiran (iD *
}

\begin{abstract}
We report the magnetization $(M)$, magnetostriction, electrical resistivity $(\rho)$, thermal conductivity $(\kappa)$ and thermopower $(S)$ of polycrystalline $\mathrm{La}_{0.9} \mathrm{Na}_{0.1} \mathrm{MnO}_{3}$ over a wide temperature range of 5 to 360 $\mathrm{K}$. This sample undergoes a paramagnetic to ferromagnetic transition around $T_{\mathrm{C}}=274 \mathrm{~K}$ and electrical resistivity $\rho$ shows an insulator-metal transition around $T_{I M}=292 \mathrm{~K}$. The sign of thermopower $S$ is positive in the entire temperature range which indicates that majority charge carriers are holes. Thermopower exhibits a peak and thermal conductivity shows a dip at $T_{C}$ in the absence of magnetic field. Large difference between the experimentally determined activation energies of $\rho$ and $S$ in the insulating state indicates small polaron hopping dominant conduction above $T_{I M}$. Polaron formation above $T_{C}$, was further confirmed from the anomaly observed in thermal expansion $\left(\Delta L / L_{0}\right)$ which shows a change in slope at $T_{I M}$. In the vicinity of $T_{C}$ at $3 T$ applied field, magneto-thermopower $(\sim 61.5 \%)$ is larger than magnetothermal conductivity $(\sim 12.7 \%)$ and magnetoresistance ( 49\%).
\end{abstract}

\section{Introduction}

Perovskites containing $\mathrm{Mn}$ ions (i.e. $\mathrm{La}_{1-x} \mathrm{~A}_{x} \mathrm{MnO}_{3}, \mathrm{~A}=\mathrm{Ca}^{2+}$, $\mathrm{Ba}^{2+}, \mathrm{Sr}^{2+}$ etc.) have been one of the hot areas of research in physics and materials chemistry because of their colossal magnetoresistance (CMR) property and its prospective applications. ${ }^{1-3}$ Researchers are intrigued by the rich distinctive physical properties of every member of the manganite-family. From a fundamental point of view, manganites show diverse phenomena such as ferromagnetism, antiferromagnetism, multiferroism, magnetically coupled or independent structural transitions, nano- meso- and micro-scale phase separation, insulator-metal (IM) transition, spin-, charge- and orbital ordering etc. .,4 $^{\mathbf{4}}$

$\mathrm{LaMnO}_{3}(\mathrm{LMO})$ containing $\mathrm{Mn}^{3+}\left(\mathrm{t}_{2 \mathrm{~g}}^{3} \mathrm{e}_{\mathrm{g}}^{1}\right)$ cations is a layered Atype antiferromagnetic (AFM) insulator with a Neel temperature $\left(T_{\mathrm{N}}\right)$ of $140 \mathrm{~K}$. Substitution with divalent alkaline earth cations $\left(\mathrm{A}=\mathrm{Ca}^{2+}, \mathrm{Sr}^{2+}\right.$ etc. $)$ at the $\mathrm{La}^{3+}$ site transforms $\mathrm{Mn}^{3+}$ into an equal amount of $\mathrm{Mn}^{4+}\left(\mathrm{t}_{2 \mathrm{~g}}^{3} \mathrm{e}_{\mathrm{g}}^{0}\right)$ and introduces ferromagnetic (FM) spin ordering for $x=\sim 0.18-0.45$ in $\mathrm{La}_{1-x} \mathrm{~A}_{x} \mathrm{MnO}_{3} .^{5}$ Ferromagnetism and metallic conduction in the divalent ion doped manganites are understood in terms of Zener's double exchange (DE) model. ${ }^{6}$ Ferromagnetic metallic state can also be induced by substitution of monovalent cations such as $\mathrm{Na}^{1+}, \mathrm{K}^{1+}$ or $\mathrm{Ag}^{1+}$ for $\mathrm{La}^{3+}$ which theoretically should introduce twice the number of holes (or $\mathrm{Mn}^{4+}$ ) in the structure compared to the

Department of Physics, Faculty of Science, National University of Singapore, 2 Science Drive 3, Singapore - 117542, Republic of Singapore.E-mail: phyrm@nus.edu.sg same concentration of divalent cation substitution and hence ferromagnetism can be obtained for a smaller amount of $\mathrm{Na}^{1+}$ or $\mathrm{K}^{1+}$ compared to any divalent ions.

Although thermal transport of hole doped LMO has been investigated in the past few years, the role of magnetic field on the thermal transport of $\mathrm{Na}^{1+}$ doped LMO system has been scarcely studied so far. Thermopower $(S)$ is sensitive to band structure and also can probe majority charge carriers. Also $S$ is less affected by the grain boundaries and hence probe intrinsic electrical conduction within grains unlike dc resistivity whose magnitude and temperature dependence is severely modified by grain boundaries. Therefore, $S$ is an effective way to study the transport properties in polycrystalline samples..$^{7-9}$ Both Shimura et al. ${ }^{10}$ and Ahmed et al. ${ }^{11}$ obtained a negative $S$ in $\mathrm{La}_{1-x} \mathrm{~A}_{x} \mathrm{MnO}_{3}$ $(x \geq 0.10)$ above ferromagnetic transition temperature, but they did not shed light on the trend of thermopower or thermal conductivity under the influence of magnetic field. Studies show that the sign of $S(T)$ can be negative or positive both reflecting holelike or electronlike transport, depending on the temperature and the degree of elemental substitution. $10 \% \mathrm{Na}$ doping is a critical composition form the point of view that in $\mathrm{Ca}^{2+}$ or $\mathrm{Sr}^{2+}$ doped compositions $S$ alters the sign with lowering temperature as $\mathrm{Mn}^{4+}$ content becomes as high as $\sim 26 \%$ (ref. 12) or $\sim 25 \%,{ }^{13}$ respectively. $\mathrm{La}_{0.9} \mathrm{Na}_{0.1} \mathrm{MnO}_{3}$ belongs to space group $R \overline{3} c$ like the parent compound with a small decrease in $\mathrm{Mn}-\mathrm{O}$ bond length and increase in $\langle\mathrm{Mn}-\mathrm{O}-\mathrm{Mn}\rangle$ angle compared to undoped LMO. ${ }^{14-16}$ A doping level exceeding 20\% changes the structure from rhombohedral to orthorhombic one. ${ }^{16}$ Considering tight-binding approximation for $\mathrm{ABO}_{3}$ structures, doping 
$\mathrm{Na}^{1+}$ at $\mathrm{La}^{3+}$ site affects charge-carrier ( $\mathrm{e}_{\mathrm{g}}$ electron) bandwidth, $W\left(\propto \frac{\cos \theta}{d_{\mathrm{Mn}-\mathrm{O}}^{3.5}}\right.$, where $\theta=180-\langle\mathrm{Mn}-\mathrm{O}-\mathrm{Mn}\rangle$ and $d_{\mathrm{Mn}-\mathrm{O}}$ is the $\mathrm{Mn}-\mathrm{O}$ bond length) advancing DE interaction in the structure. ${ }^{17}$ $W$ rises rapidly with a small increase in $\langle\mathbf{M n}-\mathrm{O}-\mathrm{Mn}\rangle$ angle or decrease in $\mathrm{Mn}-\mathrm{O}$ length favoring the evolution of the insulator-metal transition ${ }^{\mathbf{1 6}}$ which explains the enhancement of $T_{\mathrm{C}}$ by $\sim 150 \mathrm{~K}$ in only $10 \% \mathrm{Na}$ doped LMO. ${ }^{18}$

Recently, nearly $100 \%$ suppression of thermopower (negative magneto-thermopower) was reported in antiferromagnetic $\mathrm{Nd}_{0.75} \mathrm{Na}_{0.25} \mathrm{MnO}_{3} \cdot{ }^{19}$ An interesting correlation between magneto-thermopower and magnetoresistance was also reported in the same. There has been no report on combined study of thermopower, thermal conductivity and resistivity in Na-doped $\mathrm{LaMnO}_{3}$. In this work, we report simultaneous measurements of the dc electrical resistivity $(\rho)$, thermal conductivity $(\kappa)$ and thermopower $(S)$ of polycrystalline $\mathrm{La}_{0.9^{-}}$ $\mathrm{Na}_{0.1} \mathrm{MnO}_{3}$ as a function of temperature without and with an external magnetic field. In addition, magnetization, linear thermal expansion and field dependence of magnetostriction are also reported. Our investigation on temperature dependence of magnetization indicates paramagnetic (PM) to ferromagnetic transition at $T_{\mathrm{C}}=274 \mathrm{~K}$ upon cooling. Magnetic ordering is accompanied by an insulator-metal transition identified by the peak in resistivity curve at $T_{\mathrm{IM}}=292 \mathrm{~K}$. Thermopower $S$ reveals a hole doping characteristics above and below $T_{\mathrm{IM}}$. The theoretical fitting of $S(T)$ curve indicates that at the FM metallic region, diffusion; phonon drag; magnon drag all coexist whereas at low temperatures (below $80 \mathrm{~K}$ ), magnon drag dominates the thermopower value. Temperature and field dependent measurements show dominant lattice contribution to the heat conductivity $\kappa$.

\section{Experimental details}

Polycrystalline $\mathrm{La}_{0.9} \mathrm{Na}_{0.1} \mathrm{MnO}_{3}$ sample was synthesized by solidstate method using high purity $\mathrm{La}_{2} \mathrm{O}_{3}, \mathrm{MnO}_{2}$ and $\mathrm{NaCO}_{3}$ powders. Stoichiometric amount of the well mixed powders was calcined at $1000{ }^{\circ} \mathrm{C}$ for $24 \mathrm{~h}$ followed by sintering at $1150{ }^{\circ} \mathrm{C}$ for $48 \mathrm{~h}$ in few steps of intermediate grinding. The Rietveld refinement of the XRD data reveals the sample belongs to space group $R \overline{3} c$ of the rhombohedral crystal structure. Refined lattice constants of the unit cell are $a=b=5.5226 \AA$; $c=13.3481 \AA$. Magnetization measurements were performed using a Physical Property Measuring System (PPMS), equipped with a vibrating sample magnetometer. The temperature dependence of linear thermal expansion and field dependence of thermal expansion (Joule magnetostriction) were measured using a miniaturized capacitance dilatometer probe designed for PPMS. ${ }^{20}$ Four probe dc resistivity, thermopower and thermal conductivity were measured simultaneously at each stabilized temperature using the standard thermal transport option (TTO) for PPMS. In TTO, thermal conductivity $(\kappa)$ is measured directly from the applied heater power used to create temperature gradient $(\Delta T)$ between two ends of the sample. Any heat loss during the measurement is also estimated by the software and the errors due to heat loss is excluded during calculation. However, for thermopower $(S)$ the voltage drop $(\Delta V)$ across the hot and cold thermometer probes is also monitored by the TTO system and measured once a stable temperature gradient is created across these two thermometer probes due to the application of heat. PPMS served as a platform to vary temperature and magnetic field. The error for $S$ is almost negligible, whereas, for $\kappa$ it is $< \pm 0.1 \%$ at $T=10-100 \mathrm{~K}$ and $< \pm 5 \%$ at higher temperatures. Density of the pellet used for TTO measurement was estimated using Archimedes' principle which was close to $95 \%$ of its theoretical value. Magnetoresistance (MR), magnetothermal conduction (MTC) and magneto-thermopower (MTEP) at $3 \mathrm{~T}$ magnetic field of the sample is calculated using the standard relations: $\operatorname{MR}(\%)=\frac{\Delta \rho \times 100}{\rho(0)} \equiv[[(\rho(3 T)-\rho(0)) \times 100] / \rho(0)]$,

$$
\begin{gathered}
\operatorname{MTC}(\%)=\frac{\Delta \kappa \times 100}{\kappa(0)} \equiv[[(\kappa(3 T)-\kappa(0) \times 100)] / \kappa(0)] \\
\text { and } \operatorname{MTEP}(\%)=\frac{\Delta S \times 100}{S(0)} \equiv[[(S(3 T)-S(0)) \times 100] / S(0)],
\end{gathered}
$$

respectively.

\section{Results and discussion}

\subsection{Magnetic properties}

Fig. 1 shows the temperature dependent magnetization $(M)$ of polycrystalline $\mathrm{La}_{0.9} \mathrm{Na}_{0.1} \mathrm{MnO}_{3}$ (LNMO) sample measured in an applied field of $\mu_{0} H=0.1 \mathrm{~T}$. The rapid increase of magnetization at $T=T_{\mathrm{C}}$ indicates the onset of ferromagnetism. The ferromagnetic Curie temperature $T_{\mathrm{C}}=274 \mathrm{~K}$ is determined from the minimum of $\mathrm{d} M / \mathrm{d} T$ curve as shown in the same figure. The temperature variation of inverse susceptibility $(1 / \chi)$ and high temperature Curie-Weiss fit, $1 / \chi=C /\left(T-\theta_{\mathrm{W}}\right)$ of the data points are shown in the inset (a) of Fig. 1. $C$ is the Curie constant given by $C=N \mu_{0} \mu_{\mathrm{B}}^{2} \mu_{\text {eff }}^{2} / 3 k_{\mathrm{B}}$, where $N$ is the total number of PM entities per cubic meter. Best fitting above $T_{\mathrm{C}}$ in the linear region of $1 / \chi$, gives effective moment $\mu_{\text {eff }}=5.98 \mu_{\mathrm{B}}$ and Weiss temperature $\theta_{\mathrm{W}}$ of $279.3 \mathrm{~K}$ which is few kelvin higher than the

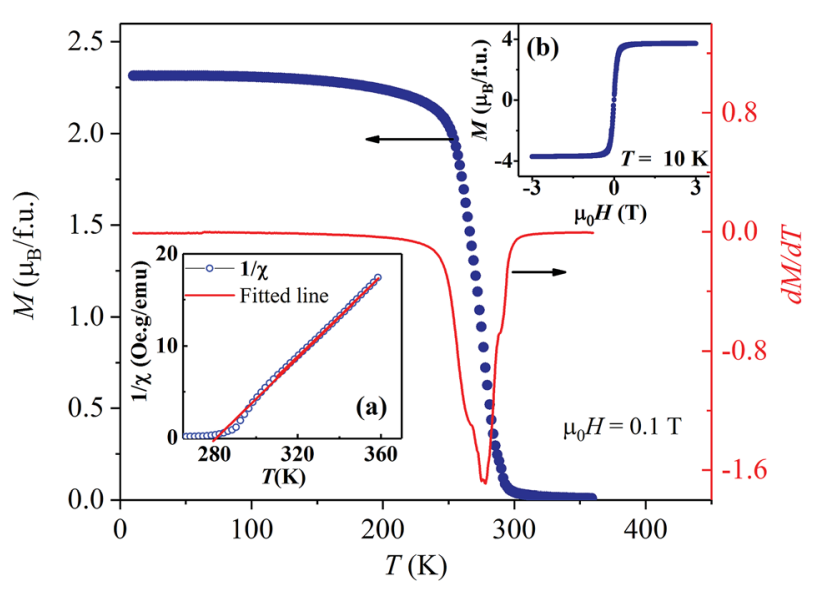

Fig. 1 Temperature dependence of magnetization $(M)$ and $d M / d T$ at an applied field $\mu_{0} H=0.1 \mathrm{~T}$, inset (a) shows inverse susceptibility vs. temperature plot, here solid line represents the Curie-Weiss fitting and (b) shows field dependent magnetization of $\mathrm{La}_{0.9} \mathrm{Na}_{0.1} \mathrm{MnO}_{3}$ at $10 \mathrm{~K}$. 
$T_{\mathrm{C}}$ obtained from $M-T$ curve. The theoretical effective magnetic moment is calculated using the equation, $\mu_{\text {eff }}=g \sqrt{S(S+1)} \mu_{\mathrm{B}}$ where, $g$ is the Lande factor and $S$ is the total spin quantum number. In $\mathrm{La}_{1-x} \mathrm{Na}_{x} \mathrm{MnO}_{3}$, as we have both $\mathrm{Mn}^{3+}$ and $\mathrm{Mn}^{4+}$ spin, the modified $S$ will be, $S_{\text {avg }}=2 x \times S^{\mathrm{Mn}^{4+}}+(1-2 x) \times S^{\mathrm{Mn}^{3+}}$. As both $\mathrm{Mn}$ ions are in the high spin state, i.e. $S^{\mathrm{Mn}^{4+}}=3 / 2$ and $S^{\mathrm{Mn}^{3+}}=2$, the experimentally estimated $\mu_{\text {eff }}$ is higher than the theoretically expected value of $4.68 \mu_{\mathrm{B}}$, considering effective moment of non-interacting $\mathrm{Mn}^{3+}$ and $\mathrm{Mn}^{4+}$ ions are $4.89 \mu_{\mathrm{B}}$ and $3.87 \mu_{\mathrm{B}}$, respectively. Enhanced $\mu_{\mathrm{eff}}$ indicates that spins are not completely independent in the PM state. It is reported that dynamical ferromagnetic nanoclusters do exists in many manganites such as in $\left(\mathrm{Sm}_{0.65} \mathrm{Sr}_{0.35}\right) \mathrm{MnO}_{3}$ and $\mathrm{Pr}_{0.65} \mathrm{Ca}_{0.25}$ $\mathrm{Ba}_{0.1} \mathrm{MnO}_{3}$ above $T_{\mathrm{C}}{ }^{21,22}$ Therefore, the above mentioned findings (high values of $\mu_{\text {eff }}$ and $\theta_{\mathrm{W}}>T_{\mathrm{C}}$ ) indicate existence of small fraction of ferromagnetic cluster above $T_{\mathrm{C}}$ in LNMO. Inset (b) of Fig. 1 shows field dependence of $M$ at $10 \mathrm{~K}$. It shows soft FM behavior (coercive field $=58.05 \mathrm{Oe}$ ) with a maximum moment of $3.7 \mu_{\mathrm{B}} / \mathrm{f}$.u at $3 \mathrm{~T}$ field, which could reach the saturation value of $3.8 \mu_{\mathrm{B}} /$ f.u expected for $20 \% \mathrm{Mn}^{4+}$ doped LMO at higher magnetic field. Hence, $10 \% \mathrm{Na}^{1+}$ substitution dopes nearly $20 \%$ of $\mathrm{Mn}^{4+}$ in the system.

\subsection{Thermal expansion properties}

Fig. 2 shows the linear thermal expansion $\left(\Delta L / L_{0}\right)$ of LNMO upon cooling from $320 \mathrm{~K}$ to $200 \mathrm{~K}$ at an external magnetic field of $\mu_{0} H=0,1$ and 3 T. $L_{0}$ is the value of $L$ at $320 \mathrm{~K}$ (normalizing temperature), the maximum operating temperature of the capacitance dilatometer. At zero field, $\Delta L / L_{0}$ decreases with lowering temperature, which indicates a contraction in length of the sample. However, $\Delta L / L_{0}$ changes slope at the onset of paramagnetic-ferromagnetic transition similar to $\mathrm{La}_{0.6} \mathrm{Y}_{0.07^{-}}$ $\mathrm{Ca}_{0.33} \mathrm{MnO}_{3} \cdot{ }^{23}$ As the external field is applied, the value of $\Delta L / L_{0}$ decreases around $T_{\mathrm{C}}$ and the change of slope also shifts towards

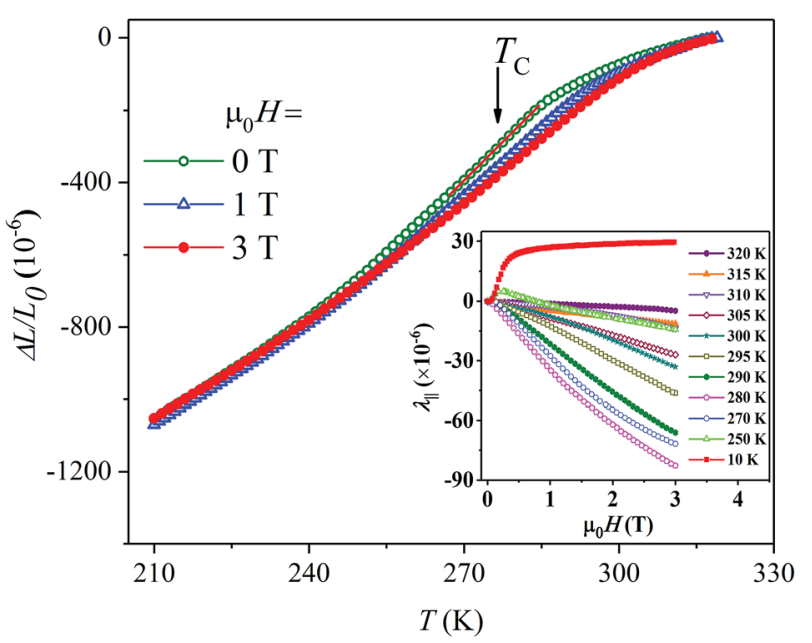

Fig. 2 Temperature dependence of thermal expansion, $\Delta L / L_{0}$ (normalizing temperature $320 \mathrm{~K}$ ) at 0,1 and $3 \mathrm{~T}$ applied fields of $\mathrm{La}_{0.9} \mathrm{Na}_{0.1} \mathrm{MnO}_{3}$ sample. Inset shows magnetostriction, $\lambda_{\|}(=[L(H)-$ $\left.L(H=0)] / L_{0}\right)$ at various temperatures. The measurement uncertainty is about $< \pm 3 \%$. The error bar is not shown for purposes of clarity. higher temperature because $T_{\mathrm{C}}$ itself increases with increasing strength of the magnetic field. The anomaly in $\Delta L / L_{0}$ around $T_{\mathrm{C}}$ can be attributed to the localization-delocalization transition of magnetic polarons as in $\mathrm{La}_{0.6} \mathrm{Y}_{0.07} \mathrm{Ca}_{0.33} \mathrm{MnO}_{3}$ (ref. 23) as well as in $\mathrm{La}_{0.67} \mathrm{Ca}_{0.33} \mathrm{MnO}_{3}{ }^{24}$ Magnetic polaron is an entity in which $\mathrm{Mn}^{3+}\left(\mathrm{d}^{4}\right)$ spins are ferromagnetically aligned locally around a $\mathrm{Mn}^{4+}\left(\mathrm{d}^{3}\right)$ ions: $\mathrm{e}_{\mathrm{g}}$ carrier hops freely within these superparamagnetic clusters. Due to Jahn-Teller nature of $\mathrm{Mn}^{3+}$, lattice distortion also accompanies local spin polarization. Localization of magnetic polarons above $T_{\mathrm{C}}$ causes extra contribution to thermal expansion, which is released when magnetic polarons collapses below $T_{\mathrm{C}}$ leading to decrease in volume. Upon application of magnetic field above $T_{\mathrm{C}}$, size of magnetic polaron expands and they start percolating which results in enhancement of $T_{\mathrm{C}}$ and shift of the thermal expansion anomaly towards higher temperature. As the magnetic field strength exceeds a critical value, the anomaly is suppressed as the crossover between the low-volume FM and high-volume PM state disappears.

The inset of Fig. 2 shows the field dependence of longitudinal magnetostriction i.e., $\lambda_{\|}\left(=[L(H)-L(H=0)] / L_{0}\right)$ at selected temperatures covering both paramagnetic and ferromagnetic regions. At $10 \mathrm{~K}, \lambda_{\|}$increases rapidly in the low magnetic fields and saturates above $0.5 \mathrm{~T}$. However, in the proximity of $T_{\mathrm{C}}(250-$ $310 \mathrm{~K}), \lambda_{\|}$decreases with increasing strength of the magnetic field and does not show saturation. $\lambda_{\|}$decreases continuously, reaches a minima of $-83 \times 10^{-6}$ near $T_{\mathrm{C}}$ with a subsequent increase for higher temperatures indicating strong spin-lattice and charge-lattice coupling at $T_{\mathrm{C}}$. The positive magnetostriction at $10 \mathrm{~K}$ is caused by increase in length (anisotropic in nature) due to spin-orbit interaction below $T_{\mathrm{C}}$. However, negative magnetostriction at and above $T_{\mathrm{C}}$ is due to the collapse of magnetic polarons.

The maximum volume magnetostriction is given by $\omega=\lambda_{\|}+$ $2 \lambda_{\perp}$, where $\lambda_{\|}$and $\lambda_{\perp}$ are the longitudinal and transverse components of magnetostriction. They are usually measured using strain gauge technique. It was not possible to measure $\lambda_{\perp}$ in our dilatometer probe as it can only measure magnetostriction along field direction, i.e. $\lambda_{\|}$. In manganites, ${ }^{23}$ magnetostriction in the PM state and close to $T_{\mathrm{C}}$ is isotropic, i.e., $\lambda_{\|} \sim \lambda_{\perp}$. Therefore, $\omega$ near $T_{\mathrm{C}}$ in LNMO can also be expressed as $\omega=3 \lambda_{\|}$ which decreases at $T_{\mathrm{C}}$ in zero field.

\subsection{Electrical and thermal transport properties}

Temperature dependence of the direct-current (DC) resistivity $(\rho)$ at $\mu_{0} H=0$ and $3 \mathrm{~T}$ magnetic fields are shown in Fig. 3(a). Upon lowering the temperature, $\rho(T, H=0 \mathrm{~T})$ initially increases as like a semiconductor and exhibits a sharp peak at $T_{\mathrm{IM}}=292$ $\mathrm{K}$ followed by metallic-like behaviour with lowering the temperature further. The insulator-metal (IM) transition temperature $T_{\mathrm{IM}}$ i.e. the position of resistivity peak is higher than $T_{\mathrm{C}}(=274 \mathrm{~K})$, determined from magnetization measurements, is similar to the report by Ye et $a l^{25}$ The IM transition temperature is also higher than $\theta_{\mathrm{W}}(=279.31 \mathrm{~K})$ estimated from the Curie-Weiss fitting with inverse magnetic susceptibility. Previous reports on similar compounds showed a broad 


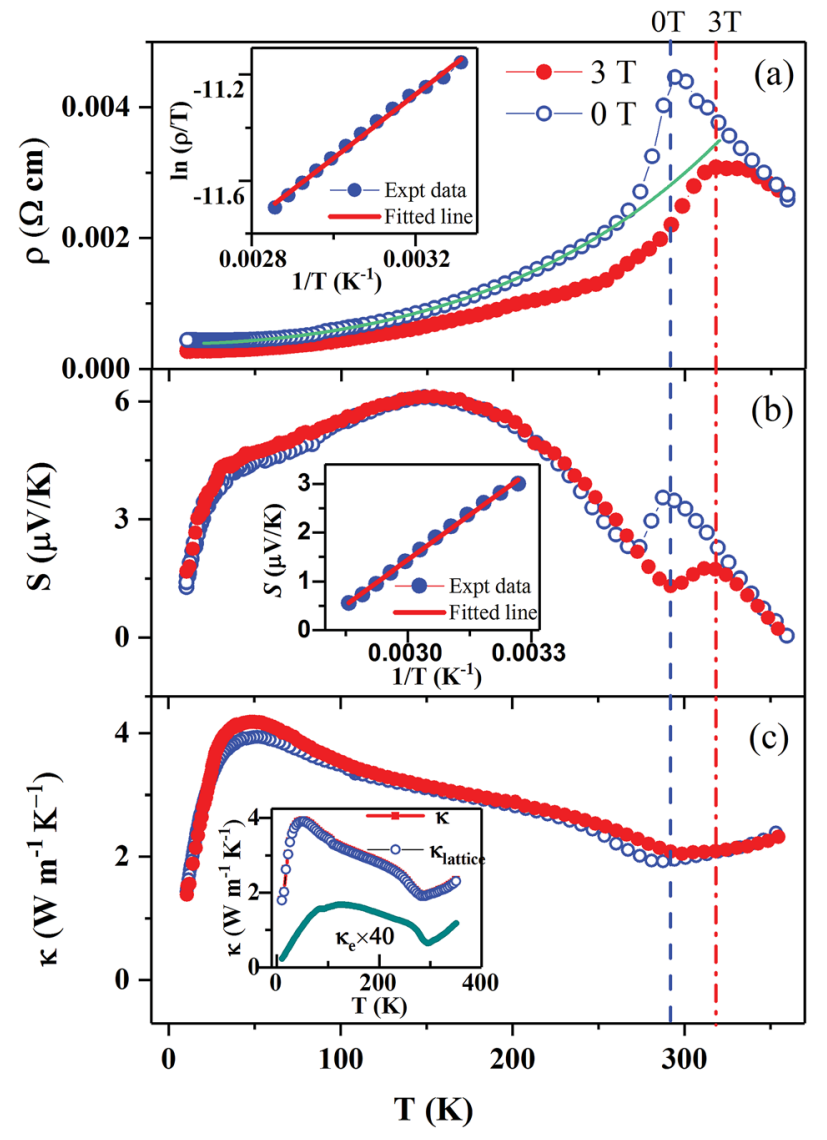

Fig. 3 Variation of (a) resistivity $\rho$ (b) thermoelectric power $S$ and (c) thermal conductivity $\kappa$ with temperature of $\mathrm{La}_{0.9} \mathrm{Na}_{0.1} \mathrm{MnO}_{3}$ at zero field (open symbol) and $3 T$ (closed symbol). The solid curve in (a) is the function $\rho(T)=\rho_{0}+A T^{2}+B T^{4.5}$. Inset (a) shows $\ln (\rho / T)$ vs. $1 / T$ plot, and the solid line is obtained by fitting the data according to the small polaron hopping model. Inset (b) includes $S$ vs. $1 / T$ plot and the solid line is a fitting based on the data. Inset (c) shows plot of $\kappa, \kappa_{\mathrm{e}}$ and $\kappa_{\text {lattice }}$ vs. temperature.

maximum below the resistivity peak due to grain boundaries, those act as regions of enhanced scattering for the conduction electrons and such maximum is absent in our sample. ${ }^{15,16,18}$ The peak value of resistivity is reduced and $T_{\mathrm{IM}}$ is shifted up under the application of magnetic field.

Fig. 3(b) and (c) show the temperature dependence of thermopower $S$ and thermal conductivity $\kappa$, respectively, measured together with dc resistivity. In zero field, sign of $S$ is positive throughout the whole temperature region, which reflects $\mathrm{e}_{\mathrm{g}}$ hole of $\mathrm{Mn}^{4+}: \mathrm{t}_{2 \mathrm{~g}}^{3} \mathrm{e}_{\mathrm{g}}^{0}$ is the majority charge carrier. Shimura et al. ${ }^{10}$ obtained a negative $S$ above $T_{\mathrm{C}}$ for $\mathrm{La}_{0.88} \mathrm{Na}_{0.09} \mathrm{Mn}_{0.96} \mathrm{O}_{3}$ and $\mathrm{La}_{0.84} \mathrm{Na}_{0.12} \mathrm{Mn}_{0.97} \mathrm{O}_{3}$ which was attributed to doping induced alteration of conduction from charge transfer type to the Motttype. In the PM state, $S(T)$ increases with lowering temperature and exhibits a peak at the IM transition and below which it decreases rapidly. However, in the FM state $S$ increases again and shows a broad maximum around $154 \mathrm{~K} . S(T)$ shows a striking resemblance with $\rho$ in the presence of an external magnetic field. Applied magnetic field lowers $S$ value prominently in the vicinity of $T_{\mathrm{IM}}$ and shifts the peak position to high temperature but it neither influences the value nor the position of the broad maximum at low temperature.

Thermal conductivity $(\kappa)$ in the paramagnetic state decreases with decreasing temperature (i.e. $\mathrm{d} \kappa / \mathrm{d} T>0$ ) which is unusual for a crystalline compound showing insulating like behavior (Fig. 3(c)) but similar to other hole-doped manganites. ${ }^{26,27} \kappa(T)$ shows a dip in the vicinity of the magnetic ordering and a few kelvins below the peak in $\rho(T)$ and $S(T)$. Within the FM state below $T_{\mathrm{C}}, \kappa(T)$ increases down to $\sim 43 \mathrm{~K}$, where it reaches a peak and below that $\kappa$ decreases rapidly. The magnitude of $\kappa$ in the metallic region varies in the range of $2-4.13 \mathrm{~W} \mathrm{~m}^{-1} \mathrm{~K}^{-1}$ which is higher than the parent compound $\left(<1 \mathrm{~W} \mathrm{~m}^{-1} \mathrm{~K}^{-1}\right.$ below room temperature $)^{28}$ but comparable with the divalent ion doped systems with similar $\mathrm{Mn}^{4+}$ concentration $\left(\kappa \sim 2.3 \mathrm{~W} \mathrm{~m}^{-1} \mathrm{~K}^{-1}\right.$ in $\left.\mathrm{La}_{0.8} \mathrm{Ca}_{0.2} \mathrm{MnO}_{3}\right){ }^{8}{ }^{8}$ The behavior of $\kappa$ in the paramagnetic region is amorphous like i.e., $\kappa$ decreases with decreasing $T$. Such characteristic of $\kappa$ is attributed to the local anharmonic lattice distortions associated with small polarons or dynamical JT distortion in A-site doped manganites. ${ }^{26}$ In general, $\kappa$ has lattice component $\kappa_{\text {lattice }}$ due to phonon vibrations, electronic component $\kappa_{\mathrm{e}}$ and the spin wave component $\kappa_{\mathrm{m}}, \kappa=\kappa_{\text {lattice }}+\kappa_{\mathrm{e}}+$ $\kappa_{\mathrm{m}}$. Above $T_{\mathrm{C}}, \kappa_{\mathrm{m}}$ is negligible and the thermal conductivity is primarily due to the other two components. Measured value of $\rho$ is used to calculate $\kappa_{\mathrm{e}}$ using the Wiedemann-Franz law, expressed by $\kappa_{\mathrm{e}}=L_{0} T / \rho$, where $L_{0}=\pi^{2} k_{\mathrm{B}}{ }^{2} / 3 \mathrm{e}^{2}$ is the Lorentz constant for metals where charge carriers behave like freeelectrons (degenerate limit). ${ }^{29}$ The estimated $\kappa_{\mathrm{e}}$ is two orders of magnitude smaller than the total $\kappa$ in the LNMO sample, which indicates phonon contribution to $\kappa$ (inset of Fig. 3(c)) is dominant than heat carried by electrical charges. Enhancement of $\kappa$ on entering the metallic state was argued due to the weakening of JT distortion and delocalization of the charge carriers. ${ }^{26}$ The pair distribution function based on the neutron scattering data shows that $\mathrm{MnO}_{6}$ octahedra in $\mathrm{La}_{1-x} \mathrm{Ca}_{x} \mathrm{MnO}_{3}(x$ $=0.2$ and 0.25 ) has uniform $\mathrm{Mn}-\mathrm{O}$ and $\mathrm{O}-\mathrm{O}$ bond lengths at low-temperature and when the temperature rises towards IM transition the disorder of $\mathrm{Mn}-\mathrm{O}$ bond lengths increases followed by the formation of small polarons. ${ }^{30}$ Reduction of $\rho$ under magnetic field should have increased $\kappa_{\mathrm{e}}$ contribution, but in the whole temperature range $\kappa_{\mathrm{e}}$ value is negligible. A dip in $\kappa(T)$ at $T_{\mathrm{C}}$ or $T_{\mathrm{N}}$ is also seen in other magnetic oxides such as $\mathrm{MnO},{ }^{31}$ and it is likely to be caused by decrease in scattering of thermal phonon by spin-fluctuations, which decreases with lowering temperature. The peak at the temperature $\sim 43 \mathrm{~K}$ is due to transition from umklapp scattering to defect dominated scattering at lower temperatures.

Fig. 4 gives a clear picture of the influence of magnetic field on the physical properties of the system. MR increases with lowering temperature in the paramagnetic state and it reaches a peak value $(\sim 49 \%)$ at $T_{\mathrm{C}}$. MR remains significant below $T_{\mathrm{C}}$ and reaches $\sim 40 \%$ at $10 \mathrm{~K}$. In the ordered state, $\rho$ rises sharply due to narrowing band width which leads to localization of charges and the formation of small polarons. Application of external magnetic field reduces the relative angle between spins which increases the electron bandwidth and enhances mobility of electrons. In addition to this, destruction of magnetic polarons under applied magnetic field gives rise to a large negative MR. 


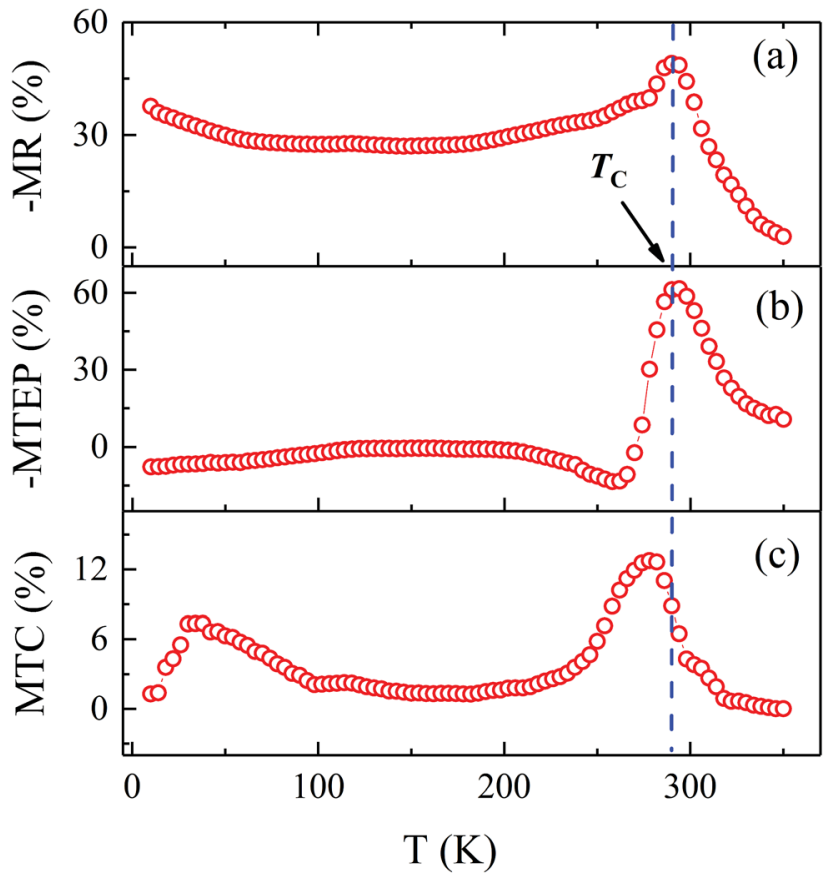

Fig. 4 (a) Plot of magnetoresistance (MR), (b) magneto-thermal conduction (MTC) and (c) magneto-thermopower (MTEP) of $\mathrm{La}_{0.9} \mathrm{Na}_{0.1} \mathrm{MnO}_{3}$.

For $T \ll T_{\mathrm{C}}$, the magnetoresistance is due to grain boundary effect: tunnelling of spin-polarized electrons between ferromagnetic grains via thin grain boundaries..$^{32}$ MTEP curve (Fig. 4(b)) reveals very interesting fact that $S$ is affected by the external field mostly around IM transition temperature, whereas $S$ in the FMM region $(T<260 \mathrm{~K})$ remains unaffected unlike $\rho(T)$. MTEP is much larger than MR in the temperature interval between $T_{\mathrm{C}}(=274 \mathrm{~K})$ and $T_{\mathrm{IM}}(=292 \mathrm{~K})$ where the spin ordering is destroyed. Unlike MR which is proportional to the density of states (DOS) at the Fermi level, MTEP depends on the asymmetry of DOS around the Fermi level due to the spin-up and spin-down states. ${ }^{33}$ Hence, in the FMM state MR is higher than MTEP but as the magnetic ordering collapses MTEP value enhances more than MR. However, MTEP observed here at comparatively low magnetic field $(61.5 \%$ at $3 \mathrm{~T})$ is comparable with the value $(\sim 80-100 \%)$ reported for antiferromagnetic $\mathrm{Nd}_{0.75} \mathrm{Na}_{0.25} \mathrm{MnO}_{3}$ system obtained at $5 \mathrm{~T} .{ }^{19}$ The magnitude of MTEP of LNMO is also close to the similar amount of hole doped $\mathrm{La}_{0.8} \mathrm{Ca}_{0.2} \mathrm{MnO}_{3}$ which shows $80 \%$ change in thermopower but at $5.7 \mathrm{~T}^{\mathrm{T}}$ magnetic field ${ }^{8}$ and single crystal $\mathrm{La}_{0.92} \mathrm{Sr}_{0.18} \mathrm{MnO}_{3}$ showing MTEP of almost $90 \%$ at 7 $\mathrm{T} .{ }^{13}$ Suppression of $S$ with magnetic field as in LNMO, is observed in few other oxides too. ${ }^{34,35}$ Yamamoto et al.$^{34}$ have shown that in the weakly ferromagnetic $\mathrm{CaRu}_{0.8} \mathrm{Sc}_{0.2} \mathrm{O}_{3}$ external magnetic field suppresses $S$, with little influence on $\rho$. They have also showed a correlation between the spin entropy and magnetothermopower. A similar explanation was also put forward by Repaka et al $^{36}$ for the room temperature ferromagnet. In antiferromagnetic $\mathrm{Nd}_{0.75} \mathrm{Na}_{0.25} \mathrm{MnO}_{3}$, a significant magnetothermopower was found above the Neel temperature even though magnetoresistance was negligible. ${ }^{19}$ However in the present study, $\rho$ and $S$ both are significantly affected by the magnetic field around $T_{\mathrm{IM}}$ (much above $T_{\mathrm{C}}$ ). It appears that $S$ is affected by the change in magnetization to a larger extend than the resistivity. A possible explanation is the decrease in the magnitude of thermopower is partly from the suppression of spin entropy. Another possibility is that the magnetic field affects the spin-dependent band structure around the Fermi level and it is reflected in magnetothermopower as mentioned before. Theoretical modelling is certainly needed to have deeper understanding.

At first sight, thermal conductivity seemed to have very small dependency on external magnetic field (Fig. 3(c)). However, measurement of $\kappa(T)$ in magnetic field makes it easier to understand the influence of spins on the heat transport property of LNMO. Temperature dependence of $\kappa$ in the hole doped manganites is attributed to two different processes; first one is considered due to the scattering of phonons by Jahn-Teller (JT) distorted $\mathrm{Mn}^{3+} \mathrm{O}_{6}$ octahedra ${ }^{26}$ and second is to the scattering of phonons by spin fluctuations. ${ }^{8}$ At $3 \mathrm{~T}$ field, reduction of spin fluctuation facilitates heat flow in the material produces a positive MTC value of about $12.7 \%$ near $T_{\mathrm{C}}$, suggesting spinphonon scattering as a decisive factor in $\kappa$ (Fig. 4(c)). Under magnetic field, suppression of the dip in $\kappa$ around $T_{\mathrm{C}}$ and marginal enhancement in conductivity can be attributed to the scattering of phonons by spin-wave. MR and MTC results unquestionably establish a strong electron-phonon-spin coupling in the Na doped LMO compound.

In manganites, high temperature transport mechanism is often analyzed using fitting of variable-range hopping or smallpolaron hopping model with the $\rho(T)$ data. However, in some cases, it is difficult to reach to a satisfactory conclusion based on the fitting results of $\rho(T)$ alone, such as in electron doped $\mathrm{La}_{0.9} \mathrm{Te}_{0.1} \mathrm{MnO}_{3}$ system ${ }^{37}$ which makes it necessary to fit the $S(T)$ data as well. In our sample, high temperature $\left(T_{\mathrm{IM}} \leq T \leq 370 \mathrm{~K}\right)$ $\rho(T)$ and $S(T)$ data fitted well with the small-polaron hopping (SPH) model, given by

$$
\rho(T)=\rho_{0} T \exp \left(E_{\rho} / k_{\mathrm{B}} T\right)
$$

and

$$
S(T)=k_{\mathrm{B}} / \mathrm{e}\left(E_{S} / k_{\mathrm{B}} T+\alpha\right),
$$

respectively, where $\rho_{0}$ and $\alpha$ are constants, $k_{\mathrm{B}}$ is the Boltzmann constant, e is the electron's charge and $E_{\rho}$ and $E_{S}$ are the activation energy values obtained from resistivity and thermopower data fitting, respectively (inset of Fig. 3(a) and (b)). We found that $E_{\rho}(103.14 \mathrm{meV})$ is an order of magnitude higher than $E_{S}$ (6.13 meV). $E_{\rho} \gg E_{S}$ is a hall mark of electrical conduction by small polaron hopping between the neighboring sites. ${ }^{38,39} E_{S}$ corresponds to the hopping energy of carriers between energetically equivalent sites with Fermi level as a reference point for the entropy, while $E_{\rho}$ contains energy to create the carriers too. Obtained value of $E_{\rho}$ agrees well with the proper stoichiometric $\mathrm{La}_{0.87} \mathrm{Na}_{0.13} \mathrm{MnO}_{3}$ compound studied by Malavasi et al. ${ }^{40}$ Thus, the electrical conduction in LNMO is governed by hopping of small polarons with the energy given by $2\left(E_{\rho}-E_{S}\right)=$ $194.02 \mathrm{meV}^{8}$ 
The thermal variation of resistivity below the upturn near $T_{\mathrm{C}}$ $(T<260 \mathrm{~K})$ is dominated by electron-electron scattering $\left(T^{2}\right.$ dependency) ${ }^{\mathbf{4 1}}$ and electron-magnon or, spin wave scattering in the ferromagnetic phase ( $T^{4.5}$ dependency). ${ }^{\mathbf{4 1 , 4 2}}$ We have fitted the present experimental data in the FMM phase using an expression of the form, $\rho(T)=\rho_{0}+A T^{2}+B T^{4.5}$ nearly perfectly as shown by the solid line in Fig. 3(a), where $\rho_{0}$ is the residual resistivity due to the temperature independent scattering processes. The fitting parameter $A\left(\sim 2.17 \times 10^{-8} \Omega \mathrm{cm} \mathrm{K}^{-2}\right)$ obtained for our samples is similar to that reported by Urushibara et al. ${ }^{\mathbf{4 1}}$ for La-Sr-Mn-O system and suggests an important role of the electron-electron scattering process below $260 \mathrm{~K}$ in the resistivity. Value of $B\left(\sim 4.73 \times 10^{-15} \Omega \mathrm{cm} \mathrm{K}^{-4.5}\right)$, related to the $T^{4.5}$ behavior suggests that electron-magnon scattering too strongly contributes to the electrical conduction in the FMM region and causes the observed trend in $\rho$ at this temperature range.

The thermopower data in the low temperature metallic region is analyzed using an equation, ${ }^{37}$

$$
S=S_{0}+S_{1} T+S_{3 / 2} T^{3 / 2}+S_{3} T^{3}+S_{4} T^{4}
$$

Here, the first term $S_{0}$ is estimated from the high temperature data extrapolation at $T=0 \mathrm{~K}$. Second term represents contribution from diffusion, the third term of the equation denotes magnon drag or the single-magnon scattering processes, while the fourth term with $T^{3}$ dependency is related to the phonon drag contribution and the last term represents the spin-wave fluctuation in the FM phase below $T_{\mathrm{C}}$. In general, $S(T)$ of manganites in FM region is analysed without considering the diffusion and phonon drag terms which was quite logical considering the position of low temperature broad peak. Phonon drag peak in thermopower is usually observed near temperature $\theta_{\mathrm{D}} / 5$, where Debye temperature $\theta_{\mathrm{D}}$ is about $320 \mathrm{~K}$ for LMO. ${ }^{43}$ Thus, the phonon drag contribution is supposed to show maximum around $60 \mathrm{~K}$ much below the observed thermopower peak here at $150 \mathrm{~K}$. To ratify further, LNMO data below $T \ll T_{\mathrm{C}}$ was fitted with equations considering (i) all the terms in eqn (3) and (ii) excluding the diffusion and phonon drag contribution terms in eqn (3). Fig. 5(a) shows a better fitting for the first case throughout a wide temperature range (49-260 K) whereas, the latter one fits the experimental data points only between $150-260 \mathrm{~K}$. Best fitting results $\left(S_{0}=5.7 \mu \mathrm{V}\right.$ $\mathrm{K}^{-1}, S_{1}=-0.116 \mu \mathrm{V} \mathrm{K}^{-2}, S_{3 / 2}=0.013 \mu \mathrm{V} \mathrm{K}^{-5 / 2}, S_{3}=-2.77 \times$ $10^{-6} \mu \mathrm{V} \mathrm{K}^{-4}$ and $S_{4}=4.06 \times 10^{-9} \mu \mathrm{V} \mathrm{K}^{-5}$ ) show $S_{3 / 2} \gg S_{3}$, suggesting that the electron-magnon scattering strongly affects the broad peak in the $S$ vs. $T$ curve but phonon drag is not negligible either. However, in the temperature range below the broad peak (80-30 K) thermopower decreases rapidly showing a $T^{3 / 2}$ dependence (inset of $4(\mathrm{a})$ ) like in other hole doped manganites. ${ }^{\mathbf{9 , 4 4}}$

Fig. 5(b) shows the figure of merit $Z T$, expressed by $Z T=\frac{S^{2} T}{\kappa \rho}$, a characteristic parameter for the thermoelectric materials. As $Z T$ has a square term of thermopower, it shows a peak around the maxima of $S(T) .10 \%$ Na doped LMO exhibits better value of $Z T$ compared to $\mathrm{La}_{0.9} \mathrm{Te}_{0.1} \mathrm{MnO}_{3}$ at room

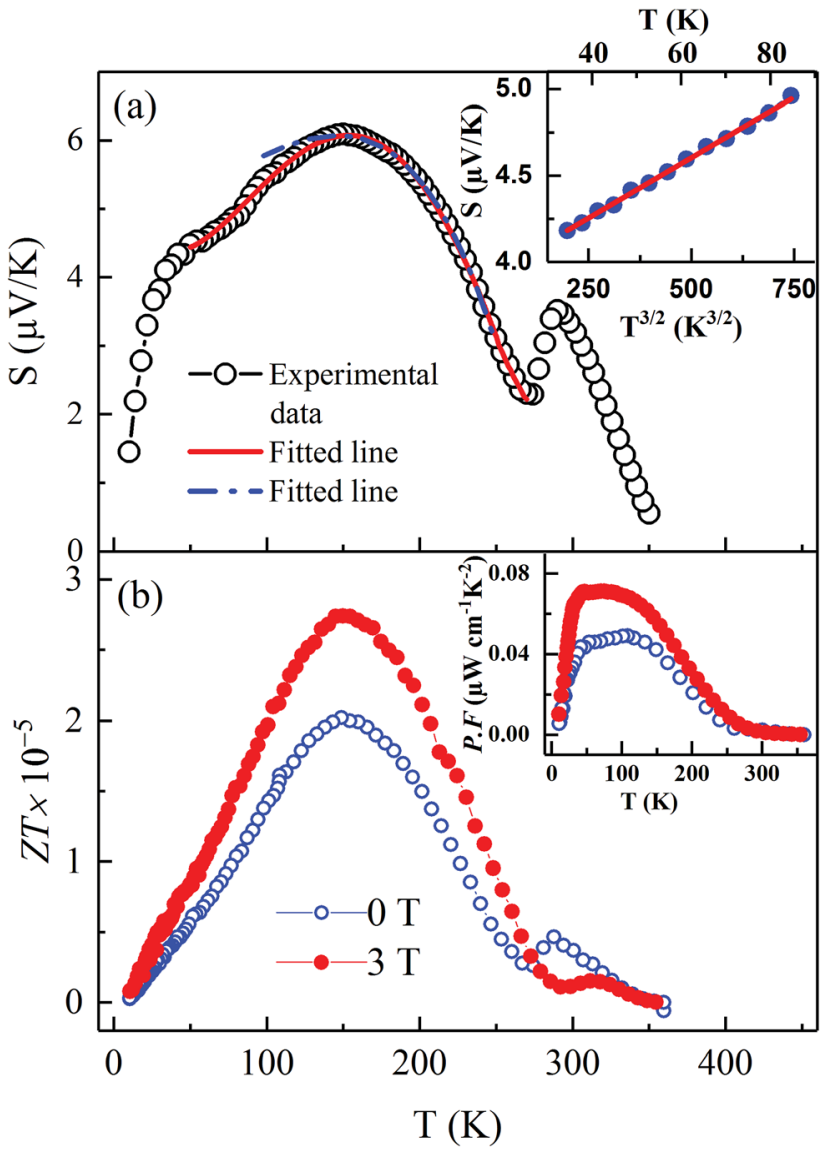

Fig. 5 (a) Temperature dependence of the thermopower for the $\mathrm{La}_{0.9} \mathrm{Na}_{0.1} \mathrm{MnO}_{3}$ sample. The solid line (red) corresponds to the expression (i) $S=S_{0}+S_{1} T+S_{3 / 2} T^{3 / 2}+S_{3} T^{3}+S_{4} T^{4}$ and dashed line (blue) represent the fitting with (ii) $S=S_{0}+S_{3 / 2} T^{3 / 2}+S_{4} T^{4}$ as discussed in the text, (b) temperature dependence of figure of merit, $Z T$ for the same sample. Inset of (a) shows $T^{3 / 2}$ dependency of $S$ at low temperature and (b) power factor $\left(S^{2} / \rho\right)$ at 0 and $3 \mathrm{~T}$ fields.

temperature $^{37}$ but it is still quite low to be useful for the practical purpose of a thermoelectric material for which $Z T \geq 1$. In the inset of Fig. 5(b), the temperature variation of power factor $\left(\right.$ P.F. $\left.=\frac{S^{2}}{\rho}\right)$ is shown. At zero and $3 \mathrm{~T}$ applied field, P.F. shows a similar trend with decreasing temperature. It starts increasing rapidly below $T_{\mathrm{C}}$, goes through a maximum value and then decreases to zero. P.F. has highest value of $0.07 \mu \mathrm{W} \mathrm{cm}{ }^{-1} \mathrm{~K}^{-2}$ around $47 \mathrm{~K}$ at $3 \mathrm{~T}$ field.

\section{Conclusions}

To conclude, effects of magnetic field on the thermal expansion, charge transport and thermal conduction mechanism for aliovalent $\mathrm{Na}^{1+}$ doped $\mathrm{LaMnO}_{3}$ were investigated. We have found anomaly in thermal expansion behavior around $T_{\mathrm{C}}$. Electrical resistivity and thermopower data analysis in the insulating region reveal that conductivity at high temperature is governed by small-polaron hopping mechanism in $\mathrm{La}_{0.9} \mathrm{Na}_{0.1} \mathrm{MnO}_{3}$. Anomaly of $\kappa$ around paramagnetic-ferromagnetic transition 
suggests coupling between thermal conductivity and magnetism. Application of magnetic field enhances thermal conductivity while suppresses the magnitude of thermopower and resistivity. The magnitude of magneto-thermopower ( $61.5 \%)$ is larger than the magnetoresistance $(\sim 49 \%)$ and magnetothermal conductivity $(\sim 12.7 \%)$.

\section{Conflicts of interest}

There are no conflicts to declare.

\section{Acknowledgements}

R. M. thanks the Ministry of Education for supporting this work (Grant no. MOE2014-T2-48/R144-000-349-112 and MOE2016-T22-098/R144-000-381-112).

\section{References}

1 C. N. R. Rao and B. Raveau, Colossal Magnetoresistance, Charge Ordering, and Related Properties of Manganese Oxides, World Scientific, Singapore, 1998.

2 J. Hu, L. Shi, Q. Liu, H. Huang and T. Jiao, Improved oxygen reduction activity on silver modified $\mathrm{LaMnO}_{3}$-graphene via shortens the conduction path of adsorbed oxygen, RSC Adv., 2015, 5, 92096.

3 H. Xu, Y. Ma, S. Zhao, W. Huang, Z. Qua and N. Yan, Enhancement of $\mathrm{Ce}_{1-x} \mathrm{Sn}_{x} \mathrm{O}_{2}$ support in $\mathrm{LaMnO}_{3}$ for the catalytic oxidation and adsorption of elemental mercury, RSC Adv., 2016, 6, 63559.

4 Y. Tokura, Critical features of colossal magnetoresistive manganites, Rep. Prog. Phys., 2006, 69, 797.

5 G. H. Jonker and J. H. Van Santen, Ferromagnetic Compounds of Manganese with Perovskite Structure, Physica, 1950, 16, 337-349.

6 C. Zener, Interaction between the d-Shells in the Transition Metals. II. Ferromagnetic Compounds of Manganese with Perovskite Structure, Phys. Rev., 1951, 82, 403.

7 A. Bhaskar, M.-S. Huang and C. -J. Liu, Effects of Fe doping on the thermal hysteresis of the $\mathrm{La}_{0.5} \mathrm{Ca}_{0.5} \mathrm{MnO}_{3}$ system, $R S C$ Adv., 2017, 7, 11543.

8 B. Chen, A. G. Rojo, C. Uher, H. L. Ju and R. L. Greene, Magnetothermal conductivity of $\mathrm{La}_{0.8} \mathrm{Ca}_{0.2} \mathrm{MnO}_{3}$, Phys. Rev. B: Condens. Matter Mater. Phys., 1997, 55, 15471.

9 P. Mandal, Temperature and doping dependence of the thermopower in $\mathrm{LaMnO}_{3}$, Phys. Rev. B: Condens. Matter Mater. Phys., 2000, 61, 14675.

10 T. Shimura, T. Hayashi, Y. Inaguma and M. Itoh, Magnetic and Electrical Properties of $\mathrm{La}_{y} \mathrm{~A}_{x} \mathrm{Mn}_{w} \mathrm{O}_{3}(\mathrm{~A}=\mathrm{Na}, \mathrm{K}, \mathrm{Rb}$, and $\mathrm{Sr}$ ) with Perovskite-Type Structure, J. Solid State Chem., 1996, 124, 250-263.

11 A. M. Ahmed, S. A. Saleh, E. M. M. Ibrahim and H. F. Mohamed, Crystal structure and some transport properties of Na-doped $\mathrm{LaMnO}_{y}$, J. Magn. Magn. Mater., 2006, 301, 452-457.

12 R. Mahendiran, S. K. Tiwary, A. K. Raychaudhuri, R. Mahesh and C. N. R. Rao, Thermopower and nature of the hole- doped states in $\mathrm{LaMnO}_{3}$ and related systems showing giant magnetoresistance, Phys. Rev. B: Condens. Matter Mater. Phys., 1996, 54, R9604.

13 A. Asamitsu, Y. Moritomo and Y. Tokura, Thermoelectric effect in $\mathrm{La}_{1-x} \mathrm{Sr}_{x} \mathrm{MnO}_{3}$, Phys. Rev. B: Condens. Matter Mater. Phys., 1996, 53, R2952.

14 M. P. Sharma, et al., Electric transport behaviour of sodiumsubstituted perovskites $\mathrm{La}_{1-x} \mathrm{Na}_{x} \mathrm{MnO}_{3}$ (for $x=0.1$ and 0.2 ) and the effect of magnetic fields, J. Phys.: Condens. Matter, 2008, 20, 425220.

15 S. Das and T. K. Dey, Structural and magnetocaloric properties of $\mathrm{La}_{1-y} \mathrm{Na}_{y} \mathrm{MnO}_{3}$ compounds prepared by microwave processing, J. Phys. D: Appl. Phys., 2007, 40, 1855.

16 S. Roy, Y. Q. Guo, S. Venkatesh and N. Ali, Interplay of structure and transport properties of sodium-doped lanthanum manganite, J. Phys.: Condens. Matter, 2001, 13, 9547.

17 P. G. Radaelli, G. Iannone, M. Marezio, H. Y. Hwang, S.-W. Cheong, J. D. Jorgensen and D. N. Argyrion, Structural effects on the magnetic and transport properties of perovskite $\mathrm{A}_{1-x} \mathrm{~A}_{x}{ }^{\prime} \mathrm{MnO}_{3}(x=0.25,0.30)$, Phys. Rev. B: Condens. Matter Mater. Phys., 1997, 56, 8265.

18 G. H. Rao, J. R. Sun, K. Bärner and N. Hamad, Crystal structure and magnetoresistance of $\mathrm{Na}$ doped $\mathrm{LaMnO}_{3}, J$. Phys.: Condens. Matter, 1999, 11, 1523.

19 D. V. Maheswar Repaka and R. Mahendiran, Giant magnetothermopower in charge ordered $\mathrm{Nd}_{0.75} \mathrm{Na}_{0.25} \mathrm{MnO}_{3}$, Appl. Phys. Lett., 2013, 103, 162408.

20 R. Küchler, T. Bauer, M. Brando and F. Steglich, A compact and miniaturized high resolution capacitance dilatometer for measuring thermal expansion and magnetostriction, Rev. Sci. Instrum., 2012, 83, 095102.

21 R. P. Borges, F. Ott, R. M. Thomas, V. Skumryev, J. M. D. Coey, J. I. Arnaudas and L. Ranno, Field-induced transition in the paramagnetic state of $\left(\mathrm{Sm}_{0.65} \mathrm{Sr}_{0.35}\right) \mathrm{MnO}_{3}$ associated with magnetic clusters, Phys. Rev. B: Condens. Matter Mater. Phys., 1999, 60, 12847.

22 A. Mleiki, R. Hanen, H. Rahmouni, N. Guermazi, K. Khirouni, E. K. Hlile and A. Cheikhrouhou, Study of magnetic and electrical properties of $\operatorname{Pr}_{0.65} \mathrm{Ca}_{0.25} \mathrm{Ba}_{0.1} \mathrm{MnO}_{3}$ manganite, RSC Adv., 2018, 8, 31755.

23 M. R. Ibarra, P. A. Algarabel, C. Marquina, J. Blasco and J. García, Large Magnetovolume Effect in Yttrium Doped La-Ca-Mn-O perovskite, Phys. Rev. Lett., 1995, 75, 3541.

24 C. Zhu, R. Zheng, J. Su and J. He, Ultrasonic anomalies in $\mathrm{La}_{0.67} \mathrm{Ca}_{0.33} \mathrm{MnO}_{3}$ near the Curie temperature, Appl. Phys. Lett., 1999, 74, 3504.

25 S. L. Ye, W. H. Song, J. M. Dai, K. Y. Wang, S. G. Wang, J. J. Du, Y. P. Sun, J. Fang, J. L. Chen and B. J. Gao, Large room-temperature magnetoresistance and phase separation in $\mathrm{La}_{1-x} \mathrm{Na}_{x} \mathrm{MnO}_{3}$ with $0.1 \leq x \leq 0.3$, J. Appl. Phys., 2001, 90, 2943.

26 D. W. Visser, A. P. Ramirez and M. A. Subramanian, Thermal Conductivity of Manganite Perovskites: Colossal Magnetoresistance as a Lattice-Dynamics Transition, Phys. Rev. Lett., 1997, 78, 3947. 
27 A. Ray and T. K. Dey, Thermal conductivity of $\mathrm{La}_{0.67}\left(\mathrm{Ca}_{x} \mathrm{Sr}_{1-x}\right)_{0.33} \mathrm{MnO}_{3} \quad(x=0,0.5, \quad 1)$ and $\mathrm{La}_{0.6} \mathrm{Y}_{0.07} \mathrm{Ca}_{0.33} \mathrm{MnO}_{3}$ pellets between 10 and $300 \mathrm{~K}$, Solid State Commun., 2003, 126, 147.

28 J. L. Cohn, J. J. Neumeier, C. P. Popoviciu, K. J. McClellan and Th. Leventouri, Local lattice distortions and thermal transport in perovskite manganites, Phys. Rev. B: Condens. Matter Mater. Phys., 1997, 56, R8495.

29 H.-S. Kim, Z. M. Gibbs, Y. Tang, H. Wang and G. J. Snyder, Characterization of Lorenz number with Seebeck coefficient measurement, APL Mater., 2015, 3, 041506.

30 S. J. L. Billinge, R. G. DiFrancesco, G. H. Kwei, J. J. Neumeier and J. D. Thompson, Direct Observation of Lattice Polaron Formation in the Local Structure of $\mathrm{La}_{1-x} \mathrm{Ca}_{x} \mathrm{MnO}_{3}$, Phys. Rev. Lett., 1996, 77, 715.

31 G. A. Slack and R. Newman, Thermal Conductivity of MnO and NiO, Phys. Rev. Lett., 1958, 1, 359.

32 R. Mahesh, R. Mahendiran, A. K. Raychaudhuri and C. N. R. Rao, Effect of particle size on the giant magnetoresistance of $\mathrm{La}_{0.7} \mathrm{Ca}_{0.3} \mathrm{MnO}_{3}$, Appl. Phys. Lett., 1996, 68, 2291.

33 M. Czerner, M. Bachmann and C. Heiliger, Spin caloritronics in magnetic tunnel junctions: ab initio studies, Phys. Rev. B: Condens. Matter Mater. Phys., 2011, 83, 132405.

34 T. D. Yamamoto, H. Taniguchi, Y. Yasui, S. Iguchi, T. Sasaki and I. Terasaki, Magneto-thermopower in the Weak Ferromagnetic Oxide $\mathrm{CaRu}_{0.8} \mathrm{Sc}_{0.2} \mathrm{O}_{3}$ : An Experimental Test for the Kelvin Formula in a Magnetic Material, J. Phys. Soc. Jpn., 2017, 86, 104707.

35 A. Kumar, C. V. Tomy and A. D. Thakur, Magnetothermopower, magnetoresistance and magnetothermal conductivity in $\mathrm{La}_{0.95} \mathrm{Sr}_{0.05} \mathrm{Co}_{1-x} \mathrm{Mn}_{x} \mathrm{O}_{3}$ $(0.00 \leq x \leq 1.00)$, Mater. Res. Express, 2018, 5, 086110.
36 D. V. Maheswar Repaka, T. S. Tripathi, M. Aparnadevi and R. Mahendiran, Magnetocaloric effect and magnetothermopower in the room temperature ferromagnet $\mathrm{Pr}_{0.6} \mathrm{Sr}_{0.4} \mathrm{MnO}_{3}$, J. Appl. Phys., 2012, 112, 123915.

37 J. Yang, Y. P. Sun, W. H. Song and Y. P. Lee, Thermopower and thermal conductivity of the electron-doped manganite $\mathrm{La}_{0.9} \mathrm{Te}_{0.1} \mathrm{MnO}_{3}$, J. Appl. Phys., 2006, 100, 123701.

38 A. J. Millis, P. B. Littlewood and B. I. Shraiman, Double Exchange Alone Does Not Explain the Resistivity of $\mathrm{La}_{1-x} \mathrm{Sr}_{x} \mathrm{MnO}_{3}$, Phys. Rev. Lett., 1995, 74, 5144.

39 M. Jaime, M. B. Salamon, K. Pettit, M. Rubinstein, R. E. Treece, J. S. Horwitz and D. B. Chrisey, Magnetothermopower in $\mathrm{La}_{0.67} \mathrm{Ca}_{0.33} \mathrm{MnO}_{3}$ thin films, Appl. Phys. Lett., 1996, 68, 1576.

40 L. Malavasi, M. Cristina Mozzati, P. Ghigna, C. B. Azzoni and G. Flor, Lattice Disorder, Electric Properties, and Magnetic Behavior of $\mathrm{La}_{1-x} \mathrm{Na}_{x} \mathrm{MnO}_{3+\delta}$ Manganites, J. Phys. Chem. B, 2003, 107, 2500-2505.

41 A. Urushibara, Y. Moritomo, T. Arima, A. Asamitsu, G. Kido and Y. Tokura, Insulator-metal transition and giant magnetoresistance in $\mathrm{La}_{1-x} \mathrm{Sr}_{x} \mathrm{MnO}_{3}$, Phys. Rev. B: Condens. Matter Mater. Phys., 1995, 51, 14103.

42 K. Kubo and N. Ohata, A Quantum Theory of Double Exchange. I, J. Phys. Soc. Jpn., 1972, 33, 21.

43 B. F. Woodfield, M. L. Wilson and J. M. Byers, LowTemperature Specific Heat of $\mathrm{La}_{1-x} \mathrm{Sr}_{x} \mathrm{MnO}_{3+\delta}$, Phys. Rev. Lett., 1997, 78, 3201.

44 M. Jaime, P. Lin, M. B. Salamon and P. D. Han, Lowtemperature electrical transport and double exchange in $\mathrm{La}_{0.67}(\mathrm{~Pb}, \mathrm{Ca})_{0.33} \mathrm{MnO}_{3}$, Phys. Rev. B: Condens. Matter Mater. Phys., 1998, 58, R5901. 